\title{
Differential expression profiles and functional analysis of circular RNAs in children with fulminant myocarditis
}

\author{
Li Zhang1 ${ }^{1}$, Bo Han*,1, Jing Wang1', Qingqing Liu', Yaru Kong ${ }^{1}$, Diandong Jiang ${ }^{1}$ \& Hailin \\ Jia ${ }^{1}$ \\ ${ }^{1}$ Department of Pediatric Cardiology, Shandong Provincial Hospital affiliated to Shandong University, No. 324 Jingwuweiqi Road, \\ Jinan, Shandong 250021, PR China \\ *Author for correspondence: hanbo35@163.com
}

\begin{abstract}
Aim: To assess differential expression profiles of circular RNAs (circRNAs) and explore their possible functions in children with fulminant myocarditis. Materials \& methods: circRNA microarray experiments were carried out for determining differential expression profiles of circRNAs in three children with fulminant myocarditis and three healthy volunteers. Functional analysis and circRNA-miRNA-mRNA interaction network building were conducted to study biological functions. Results: This work identified 2281 upregulated and 892 downregulated circRNAs. Further assessment confirmed hsa_circ_0071542 upregulation (2.5-fold) in fulminant myocarditis. Functional analysis demonstrated the differentially expressed circRNAs mainly contributed to inflammation and immunity. Conclusion: circRNAs might have substantial roles in pediatric fulminant myocarditis, and hsa_circ_0071542 could serve as a promising biomarker.
\end{abstract}

First draft submitted: 23 April 2019; Accepted for publication: 24 May 2019; Published online: 14 June 2019

Keywords: circular RNA • fulminant myocarditis • functional analysis • hsa_circ_0071542 • microarray

Myocarditis represents myocardial inflammation caused by diverse etiologies; it leads to heart dysfunction and involves reduced systolic and/or diastolic functions, as well as arrhythmias [1]. Fulminant myocarditis, the most serious type of myocarditis, is featured by a rapid disease onset and dramatically accelerated progression, causing highly damaging heart failure, severe alterations in hemodynamics and cardiogenic shock [2]. Pulmonary, hepatic and renal dysfunctions might develop concomitantly or at a later time because of low perfusion [3]. The pathogenesis of myocarditis remains unclear, while fulminant myocarditis involves direct myocardial (infection) and indirect (immunologic response and overwhelming inflammatory reactions) injuries [4]. It is rather challenging to diagnose myocarditis in pediatric cases, as clinical manifestations range from no myocardial dysfunction to sudden cardiac death [5]. The mortality of fulminant myocarditis in children is high [6]. A deep exploration of its pathogenesis is therefore significant, to accomplish early diagnosis and identify new treatment targets.

Circular RNAs (circRNAs) are noncoding RNAs obtained by back-splicing of precursor mRNAs from exons of multiple eukaryotic genes [7]. There are several known biological characteristics of circRNAs. First, circRNAs are closed loop structures showing no $5^{\prime}-3^{\prime}$ polarity or polyadenylation, conferring them resistance to RNA exonucleases and increased stability compared with linear RNAs [8]. In addition, circRNAs are lowly expressed in general, often exhibiting cell-type- and tissue-specificities [9]. The biological functions of circRNAs remain largely elusive; however, the reported roles comprise microRNA or protein sequestration, transcriptional regulation, interference with splicing and translation into polypeptides [10]. Importantly, accumulating evidence suggests circRNAs could constitute competitive endogenous RNAs (ceRNAs) of miRNAs via miRNA response elements (MREs), competitively binding miRNAs for gene regulation [11]. For instance, Changa et al. reported an oncogenic function for hsa_circ_0026134 in non-small-cell lung cancer cells via miR-1256 and miR-1287 sponging [12]. Meanwhile, the circRNA Atp9b was reported by Zhou et al. to regulate osteoarthritis progression through miR$138-5 p$ targeting $[13]$.

Future Medicine 
Table 1. Detailed information about the fulminant myocarditis (M) and normal control (N) groups.

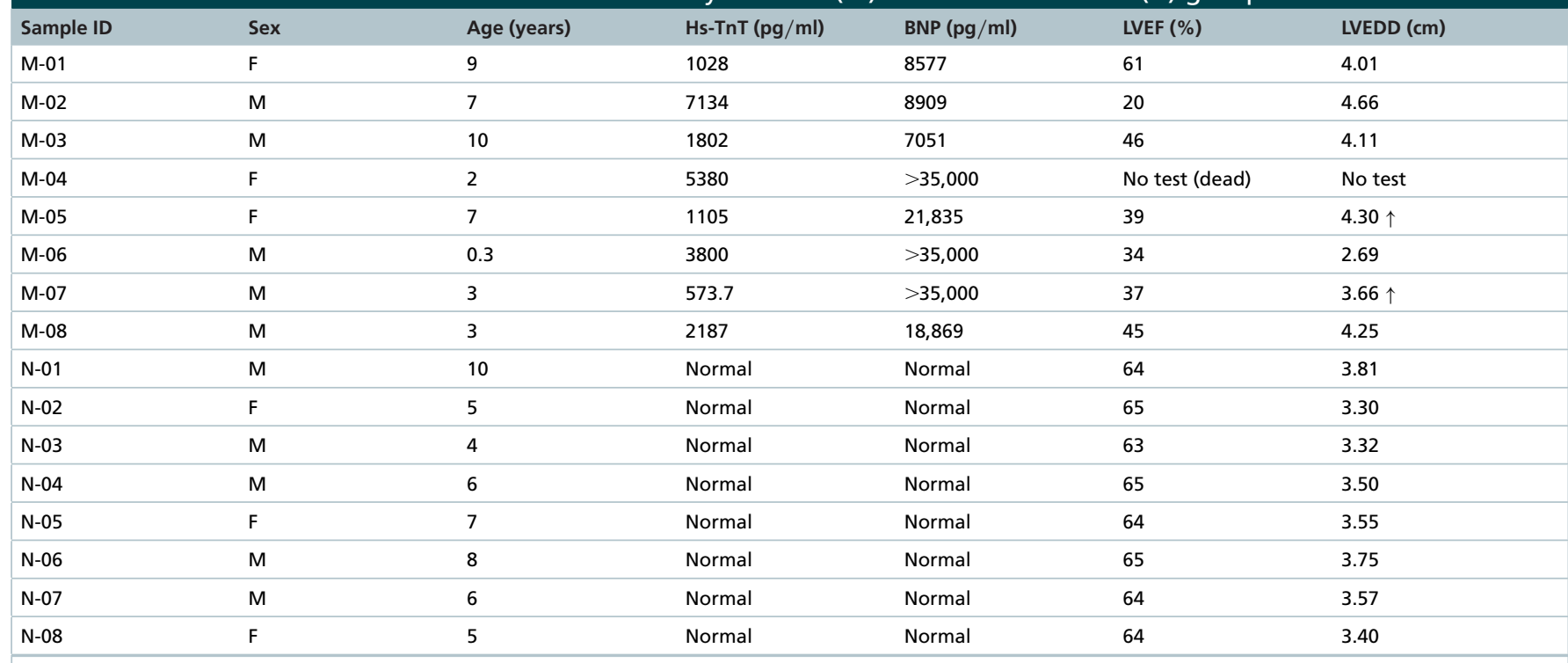

$\uparrow$ : Larger than normal.

BNP: Brain natriuretic peptide, an index of heart failure (normal range, 0-450 pg/ml); F: Female; Hs-TnT: Hypersensitive troponin T, a myocardial injury marker (normal range, 3-14 $\mathrm{pg} / \mathrm{ml}) ; \%) ;$ LVEDD: Left ventricular end-diastolic dimension, tested by echocardiography; LVEF: Left ventricular ejection fraction, tested by echocardiography, indicating cardiac contraction ability (normal value >60; M: Male.

circRNAs have been recognized as imperative regulators in several human diseases [14], including cancer, neurological disorders and cardiovascular disease [15]. Emerging evidences indicate that circRNAs are not only implicated in various cardiovascular diseases [16] but also constitute intracellular effectors causing pathophysiological alterations in cardiovascular tissues [17,18]. Moreover, a number of circRNAs control immune and inflammatory reactions [19,20]. Nevertheless, the expression patterns and roles of circRNAs in fulminant myocarditis remain undefined. In this work, we hypothesized that circRNAs are involved in fulminant myocarditis.

To test the above hypothesis, the expression patterns of circRNAs in leukocytes separated from peripheral blood samples of three children with fulminant myocarditis and three healthy volunteers were assessed by circRNA microarray analysis. Then, seven select differentially expressed circRNAs were further assessed by quantitative real time-PCR (RT-qPCR); Hsa_circ_0071542 was confirmed to be significantly upregulated by 2.5 -fold in children with fulminant myocarditis $(\mathrm{p}<0.05)$. This study provides a novel basis for substantial roles of circRNAs in fulminant myocarditis, and the above findings suggest hsa-circ-0071542 might constitute a potential biomarker of fulminant myocarditis.

\section{Materials \& methods}

Patients \& peripheral blood specimens

Peripheral blood samples from eight children with fulminant myocarditis and eight healthy volunteers were obtained at the Department of Pediatric Cardiology, Jinan hospital (China). The samples were collected between 1 October 2018 and 28 February 2019. All fulminant myocarditis cases were clinically diagnosed according to the Chinese society of cardiology expert consensus criteria [3]. Exclusion criteria were as follows: other cardiovascular diseases such as congenital heart disease, dilated or hypertrophic cardiomyopathy, and arrhythmia not related to fulminant myocarditis; congenital/acquired autoimmune pathologies and other immune diseases; and fulminant myocarditis treated with glucocorticoids or immunosuppressants before specimen collection. Control patients were healthy volunteers with age and gender matched with those of fulminant myocarditis cases. Basic information of both groups and clinical presentations of fulminant myocarditis patients are presented in Table 1 and Table 2, respectively. Besides, three pairs of samples were used for microarray detection, and eight pairs (including the three pairs used for microarrays) were assessed by RT-qPCR for microarray data verification. This study had approval from the institutional Ethics Committee, and the parents of each patient ( $<18$ years old) provided signed informed consent for research purpose. 
Table 2. Clinical presentations of fulminant myocarditis patients.

\begin{tabular}{|c|c|c|c|c|c|}
\hline Sample & Symptoms at onset & ECG & BP $(\mathrm{mmHg})$ & Phenotypes & Assisted circulation \\
\hline M-01 & Repeated syncope & $11 I^{\circ}$ AVB & $92 / 64$ & Adams-Strokes syndrome & Temporary pacemaker \\
\hline M-02 & $\begin{array}{l}\text { Headache, stomach ache, } \\
\text { emesis }\end{array}$ & $1^{\circ} \mathrm{AVB}$ & $86 / 60$ & $\begin{array}{l}\text { Acute cardiac } \\
\text { insufficiency }\end{array}$ & ECMO \\
\hline M-03 & $\begin{array}{l}\text { Fever, headache, emesis, } \\
\text { chest pain, chest distress }\end{array}$ & ST-T change & $80 / 51$ & Acute heart failure & None \\
\hline M-04 & $\begin{array}{l}\text { Fever, stomachache, } \\
\text { dyspnea }\end{array}$ & VT, VF & $72 / 35^{\dagger}$ & $\begin{array}{l}\text { Acute heart failure } \\
\text { Cardiac shock }\end{array}$ & Death \\
\hline M-05 & $\begin{array}{l}\text { Fever, cough, emesis, } \\
\text { chest pain, weak, poor } \\
\text { spirit }\end{array}$ & $\mathrm{III}^{\circ}$ AVB & $77 / 53$ & $\begin{array}{l}\text { Acute heart failure } \\
\text { Cardiac shock }\end{array}$ & Temporary pacemaker \\
\hline M-06 & $\begin{array}{l}\text { Fever, convulsion, poor } \\
\text { appetite, poor spirit }\end{array}$ & Inverted T wave & Undetectable $e^{\ddagger}$ & $\begin{array}{l}\text { Acute heart failure } \\
\text { Cardiac shock }\end{array}$ & ECMO \\
\hline M-07 & Cough, poor spirit, weak & $\mathrm{III}^{\circ}$ AVB & $75 / 45$ & $\begin{array}{l}\text { Acute heart failure } \\
\text { Cardiac shock }\end{array}$ & Temporary pacemaker \\
\hline$M-08$ & Fever, emesis, convulsion & $\mathrm{III}^{\circ}$ AVB & Undetectable $\ddagger$ & $\begin{array}{l}\text { Adams-Strokes syndrome } \\
\text { Cardiac shock }\end{array}$ & Temporary pacemaker \\
\hline
\end{tabular}

†The blood pressure was maintained by administering dopamine and isoprenaline.

$\ddagger$ Blood pressure may be undetectable with hypotension severe enough.

AVB: Atrioventricular block; BP: Blood pressure at admission; ECG: Electrocardiograph; ECMO: Extracorporeal membrane oxygenation; VF: Ventricular fibrillation: VT: Ventricular tachycardia.

\section{RNA extraction \& quality control}

After separation of leukocytes from the peripheral blood samples (three children with fulminant myocarditis and three healthy volunteers), total RNA from the isolated leukocytes was extracted with RNeasy Total RNA Isolation Kit (Qiagen, Germany) as directed by the manufacturer. Total RNA was assessed for RNA integrity number (RIN) on an Agilent Bioanalyzer 2100 (Agilent Technologies, CA, USA) or electrophoretically (denaturing agarose gel). A NanoDrop ND- 2000 spectrophotometer (NanoDrop, DE, USA) was employed to evaluate total RNA amounts and purity. The presence of genomic DNA was ruled out using an Agilent Bioanalyzer 2100. Qualified RNA was employed in microarrays and RT-qPCR.

\section{circRNA microarray analysis}

Qualified RNA samples were employed for producing biotinylated cRNA targets with Sino Human ceRNA array V3.0 (Shanghai Sinomics Corporation, China). Upon hybridization of the biotinylated cRNA targets, an Agilent Microarray Scanner (Agilent Technologies) was used for slide scanning. Data extraction was carried out with the Feature Extraction software 10.7 (Agilent Technologies). The Quantile algorithm of the LIMMA package in R was employed for raw data normalization. Data analysis was carried out as directed by Agilent Technologies at Sinotech Genomics Corporation. A fold change cutoff of two was adopted.

\section{Functional analysis of differentially expressed circRNAs}

To assess their roles, we predicted the MREs of differentially expressed circRNAs and constructed a circRNAmiRNA-mRNA network with the Arraystar miRNA target prediction software (Arraystar, MD, USA). Meanwhile, gene ontology (GO) and Kyoto encyclopedia of genes and genomes (KEGG) pathway enrichment analyses were undertaken for constructing a significant annotation of genes by Fisher's exact test with 'clusterProfiler' package in the R software.

\section{Quantitative real time-PCR}

RT-qPCR was carried out to quantitate the levels of five upregulated (has_circ_0070186, has_circ_0014350, has_circ_0071542, has_circ_0073748, and has_circ_0072017) and two downregulated (has-circ-0069387 and hascirc-0073029) circRNAs on a LightCycler480 system (Roche Diagnostics, Switzerland). Extracted RNA was subjected to cDNA synthesis with PrimeScript ${ }^{T M}$ RT reagent Kit (Takara, China). RT-qPCR was conducted with TB Green ${ }^{T M}$ Premix Ex Taq ${ }^{T M}$ II (Takara) as directed by the manufacturer. Primers are shown in Table 3, and were synthesized by BioSune Co., Ltd. (China). circRNA levels were normalized to ACTB expression. The relative expression levels were determined by the $2^{-\Delta \Delta \mathrm{Ct}}$ method. 
Table 3. Specific primers for quantitative real time-PCR

\begin{tabular}{|c|c|c|}
\hline Primer name & Primer sequence $\left(5^{\prime}-3^{\prime}\right)$ & Length (bp) \\
\hline has_circ_0014350 & $\begin{array}{l}\text { F: 5' TTTGCTCCTGTTCTGTCACTT 3' } \\
\text { R: } 5^{\prime} \text { TGTCCCAGACTTGTAGTTTGAT } 3^{\prime}\end{array}$ & 126 \\
\hline has_circ_0069387 & $\begin{array}{l}\text { F: } 5^{\prime} \text { CAGATCAGCTCTTGCTTTTATT } 3^{\prime} \\
\text { R: } 5^{\prime} \text { GAGAATCACCGTTTCATAAT } 3^{\prime}\end{array}$ & 156 \\
\hline has_circ_0070186 & $\begin{array}{l}\text { F: } 5^{\prime} \text { GGGCATTTTGAAGACTTACTG } 3^{\prime} \\
\text { R: } 5^{\prime} \text { ATGGATTTCTTTAGCTGCTTT } 3^{\prime}\end{array}$ & 200 \\
\hline has_circ_0071542 & $\begin{array}{l}\text { F: } 5^{\prime} \text { AGGCTCAGCACTGATCCAGAA } 3^{\prime} \\
\text { R: } 5^{\prime} \text { GAAATACACCAAGGGCTCGTC } 3^{\prime}\end{array}$ & 135 \\
\hline has_circ_0073748 & $\begin{array}{l}\text { F: } 5^{\prime} \text { CTGAAGAAGAGGAGGAGGAGG } 3^{\prime} \\
\text { R: } 5^{\prime} \text { ACCAAGTGCAGTAGCAAGAGG 3' }\end{array}$ & 171 \\
\hline has_circ_0072017 & $\begin{array}{l}\text { F: 5' CGCCAGAAAACTACCACTACTTG 3' } \\
\text { R: } 5^{\prime} \text { CTTGGAGGTGAGCAGTCCCG 3' }\end{array}$ & 128 \\
\hline ACTB & $\begin{array}{l}\text { F: 5' AGTTGCGTTACACCCTTTCTTG 3' } \\
\text { R: } 5^{\prime} \text { CACCTTCACCGTTCCAGTTTT } 3^{\prime}\end{array}$ & 149 \\
\hline
\end{tabular}

Statistical analysis

SPSS 24.0 (SPSS, IL, USA) and GraphPad Prism 7.0 (GraphPad Software, CA, USA) were employed for data analysis. Data are mean \pm standard deviation, and comparisons were performed by Student's $t$-test. All assays were carried out three-times or more. A p-value $<0.05$ indicated statistical significance.

\section{Results}

Expression profiles of circRNAs in children with fulminant myocarditis

In this work, 53635 circRNAs were identified in three children of fulminant myocarditis and three healthy volunteers by circRNA microarray analysis. A volcano plot revealed differentially expressed circRNAs with various p-values and fold changes between the case and control groups (Figure 1A). Hierarchical clustering analysis illustrated that circRNA expression amounts could be distinguished in the related heat map (Figure 1B).

As shown in Supplementary File 1, 3173 circRNAs showed differential expression in fulminant myocarditis cases in comparison with control patients (fold change $>2, p<0.05$ ), including 2281 upregulated and 892 downregulated circRNAs. The ten most upregulated and ten most downregulated circRNAs according to fold change are listed in Table 4. Additionally, 733 upregulated and 1437 downregulated mRNAs were detected in the fulminant myocarditis group in comparison with the control group (Supplementary File 2). The distribution of the detected differentially expressed circRNAs in human chromosomes is depicted in Figure 1C. The circRNAs with altered levels mainly derived from Chr 1 (12.29\%; 390/3173), Chr 2 (7.97\%; 253/3173) and Chr 4 $(7.41 \% ; 235 / 3173)$ in descending order. It was also revealed that more upregulated circRNAs were found than the downregulated ones in every chromosome. Moreover, the lengths of the differentially expressed circRNAs were often less than 2000 nucleotides (Figure 1D).

\section{Construction of a circRNA-miRNA-mRNA interaction network}

Previous findings [11] suggest circRNAs act as miRNA sponges by binding MREs in a competitive manner, relieving the inhibitory effects of miRNAs on target genes and increasing target gene amounts. Interactions of circRNAs with putative complementary miRNAs were assessed with Cytoscape 3.5, and five high-binding potential miRNAs for each circRNA are shown (Supplementary File 3). The network depicting the top ten upregulated and ten downregulated circRNAs with respective miRNA targets are displayed in Figure $2 \mathrm{~A}$.

We selected seven differentially expressed circRNAs according to the functions of the respective differentially expressed mRNAs, which were regulated by competitively binding the MREs of the corresponding miRNAs. These differentially expressed mRNAs, including AKT3, FOS, MAPK14, CD3D, IL1ORB and JUNB, are involved in T-cell receptor, Janus kinase-signal transducers and activators of transcription (JAK-STAT), and TNF signaling pathways. All the above mRNAs and pathways are related to immunity and inflammation [21-23], contributing to fulminant myocarditis. Detailed information about the seven selected circRNAs and the corresponding miRNAs and target genes is shown in Table 5. Then, a circRNA-miRNA-mRNA network encompassing these seven circRNAs was 
(A)

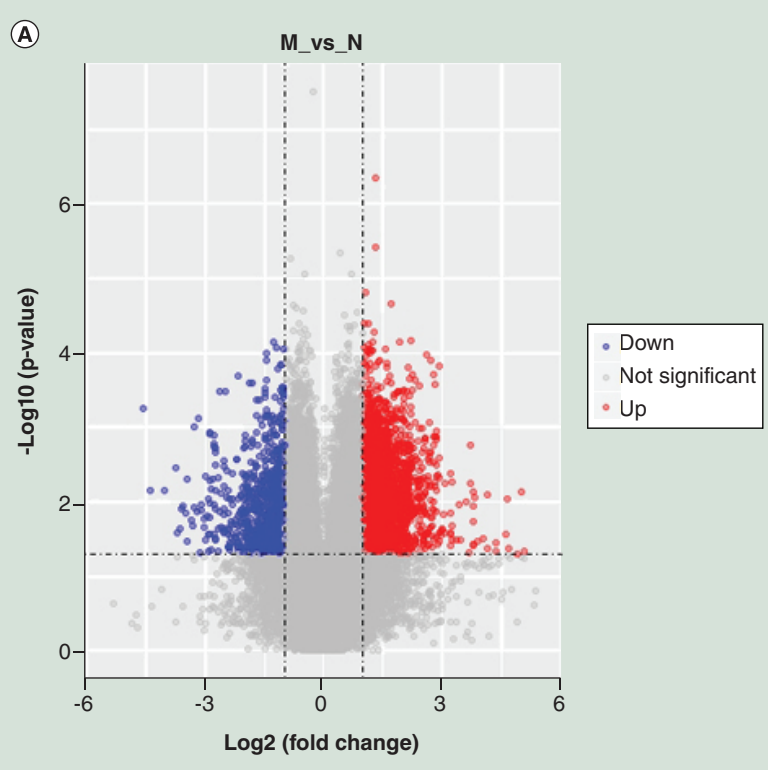

(C)

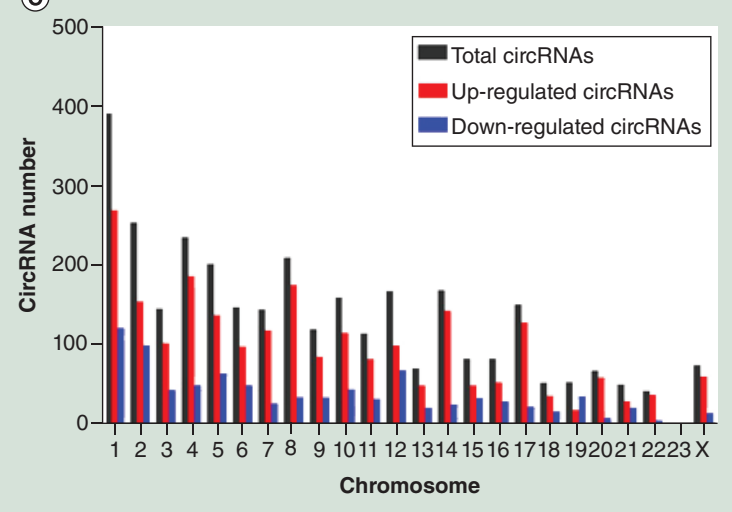

(B)

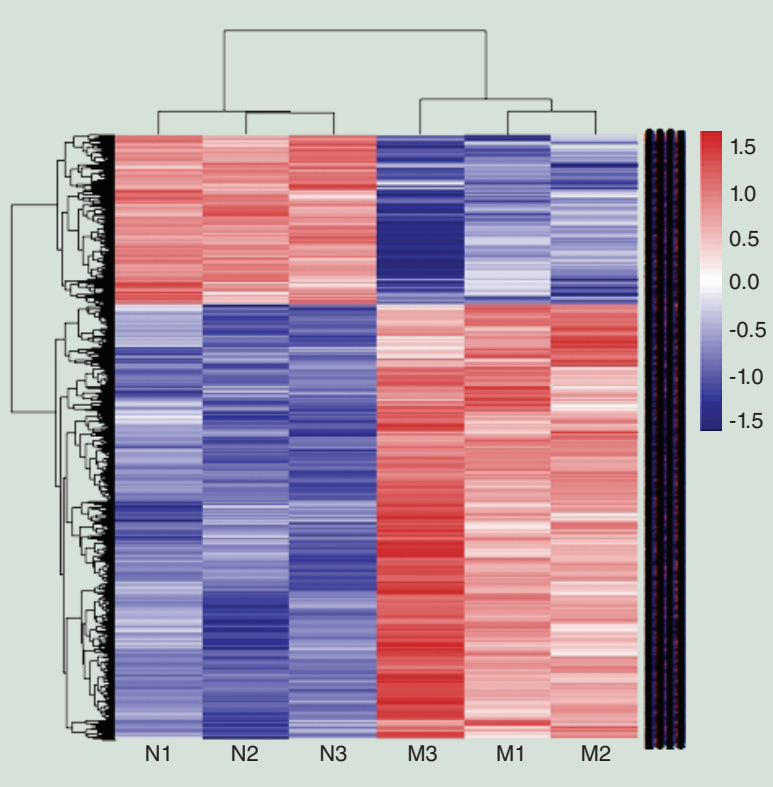

(D)

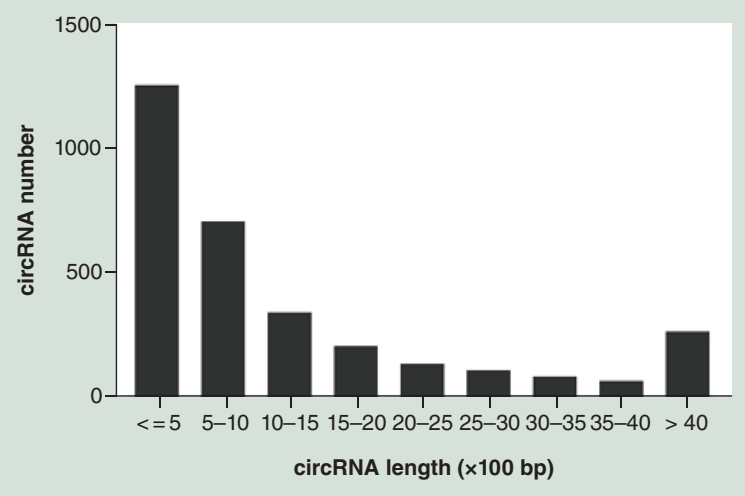

Figure 1. Expression levels of circular RNAs (circRNA) in fulminant myocarditis. (A) A volcano plot showing differentially expressed circRNAs with various $p$-values and fold changes. Horizontal line, $p$-value $=0.05$ (-log10 scaled); vertical line, fold change $=2$ (log2 scaled). Red points, upregulated circRNAs; blue points, downregulated circRNAs. (B) Hierarchical cluster analysis of circRNAs with altered expression ( $p<0.05$, fold change $>2$ ) between the two groups. Red strip, high relative expression; blue strip, low relative expression; white strip, no change in gene expression. Color brightness reflects the degree of expression increase or decrease. (C) Distribution of differentially expressed circRNAs in human chromosomes. (D) Length distribution of all differentially expressed circRNAs. circRNA: Circular RNA.

generated to visualize the interactions of circRNAs, miRNAs and target genes with the Cytoscape 3.5 software (Figure 2B).

\section{Validation of circRNA expression}

The expression levels of the seven selected (five upregulated and two downregulated) circRNAs with altered amounts were validated by RT-qPCR. Hsa_circ_0072017 failed in RT-qPCR analysis with nonspecific amplification. Meanwhile, the expression trends of two circRNAs (hsa_circ_0014350 and hsa_circ_0073029) were inconsistent with microarray data (Figure 3). Among the remaining four circRNAs, hsa_circ_0071542 was confirmed to be upregulated by 2.5 -fold in the fulminant myocarditis group versus control patients $(\mathrm{p}<0.05)$, while hsa_circ_0073748, hsa_circ_0070186 and hsa_circ_0069387 level changes lost statistical significance ( $\mathrm{p}>0.05)$. Relative hsa_circ_0071542 amounts were different in the eight fulminant myocarditis patients assessed, with patient M-04 displaying the highest expression (Figure 3C). 


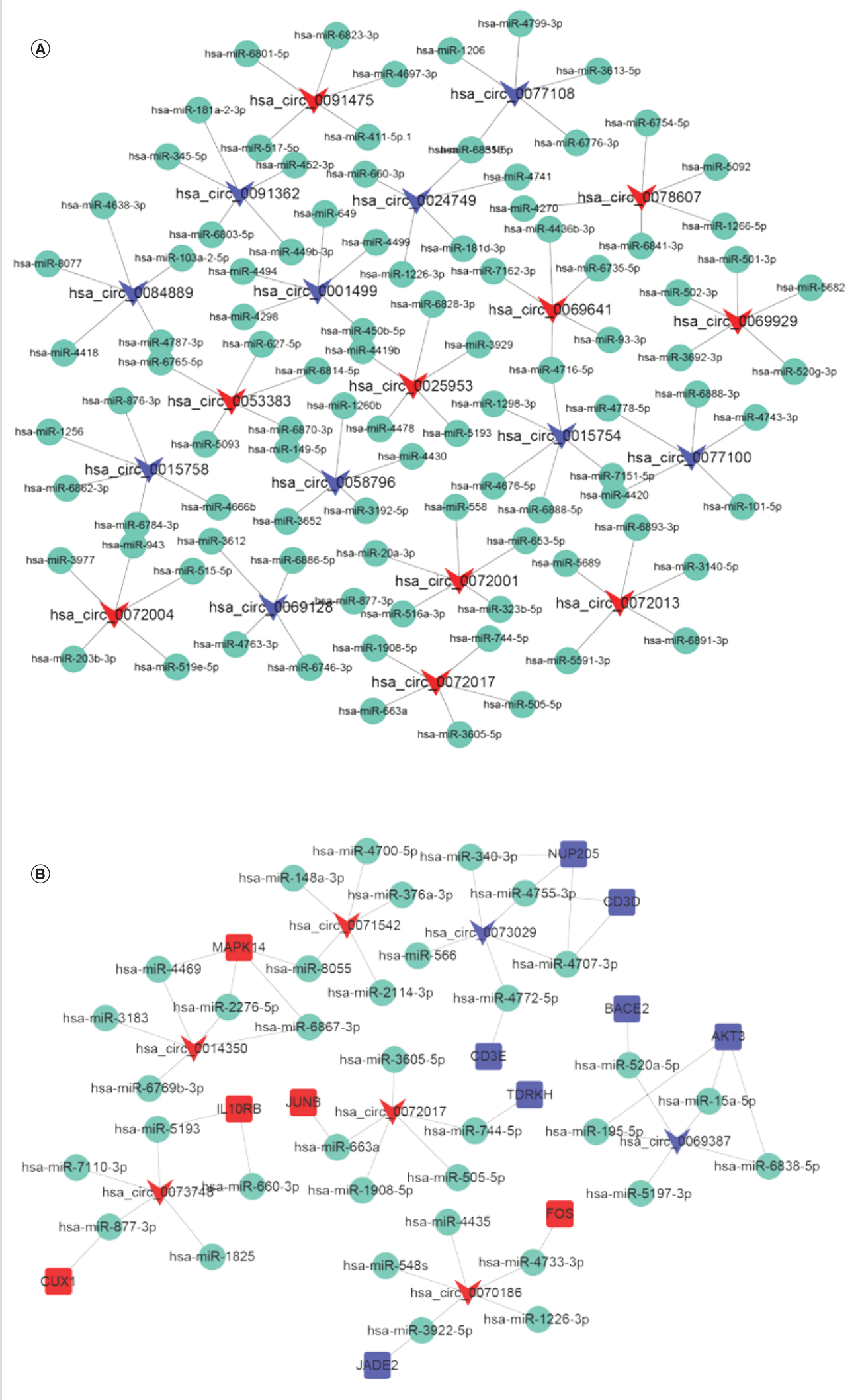

Figure 2. Circular RNA-miRNA-mRNA interaction network. (A) circRNA-miRNA interaction network of the ten most upregulated and downregulated circRNAs. (B) circRNA-miRNA-mRNA interaction network of seven selected circRNAs. Inverted triangles represent circRNAs; ellipse nodes represent the predicted miRNAs; rectangles represent mRNAs (red, upregulated; blue, downregulated). circRNA: Circular RNA. 
Table 4. Serial numbers, p-values, fold changes, chromosomes of origin, sequence lengths and host genes of the top ten upregulated and ten downregulated circular RNAs associated with fulminant myocarditis.

\begin{tabular}{|c|c|c|c|c|c|c|}
\hline circRNA & p-values & Fold change & Regulation & Chromosome & Seq-length & Host gene \\
\hline hsa_circ_0072013 & 0.047285 & 33.94341 & Up & chr5 & 315 & MY010 \\
\hline hsa_circ_0069929 & 0.007465 & 32.61925 & Up & chr4 & 4300 & ADAMTS3 \\
\hline hsa_circ_0072001 & 0.049728 & 30.16026 & Up & chr5 & 190 & MYO10 \\
\hline hsa_circ_0072004 & 0.042864 & 25.65144 & Up & chr5 & 750 & MY010 \\
\hline hsa_circ_0053383 & 0.009305 & 24.98148 & Up & chr2 & 185 & GALNT14 \\
\hline hsa_circ_0078607 & 0.027729 & 24.66401 & Up & chr6 & 546 & $S L C 22 A 3$ \\
\hline hsa_circ_0025953 & 0.047409 & 20.50623 & Up & chr12 & 4217 & ARID2 \\
\hline hsa_circ_0072017 & 0.036405 & 20.40538 & Up & chr5 & 1398 & MYO10 \\
\hline hsa_circ_0091475 & 0.043338 & 17.75699 & Up & $\operatorname{chrX}$ & 318 & $O D Z 1$ \\
\hline hsa_circ_0069641 & 0.00803 & 17.5466 & Up & chr4 & 958 & CORIN \\
\hline hsa_circ_0077108 & 0.00055 & 23.94731 & Down & chr6 & 209 & MYO6 \\
\hline hsa_circ_0024749 & 0.007165 & 20.90624 & Down & chr11 & 433 & FEZ1 \\
\hline hsa_circ_0084889 & 0.007148 & 16.39464 & Down & chr8 & 287 & TMEM67 \\
\hline hsa_circ_0001499 & 0.003571 & 13.61941 & Down & chr5 & 338 & None \\
\hline hsa_circ_0015754 & 0.026805 & 13.28069 & Down & chr1 & 986 & $\mathrm{CFH}$ \\
\hline hsa_circ_0015758 & 0.023569 & 12.54721 & Down & chr1 & 537 & $\mathrm{CFH}$ \\
\hline hsa_circ_0058796 & 0.012563 & 12.18611 & Down & chr2 & 654 & $A G A P 1$ \\
\hline hsa_circ_0091362 & 0.011464 & 11.95163 & Down & chrX & 1195 & WDR44 \\
\hline hsa_circ_0077100 & 0.013999 & 11.18568 & Down & chr6 & 436 & MYO6 \\
\hline hsa_circ_0069128 & 0.034942 & 11.06287 & Down & chr4 & 2064 & $A F A P 1$ \\
\hline
\end{tabular}

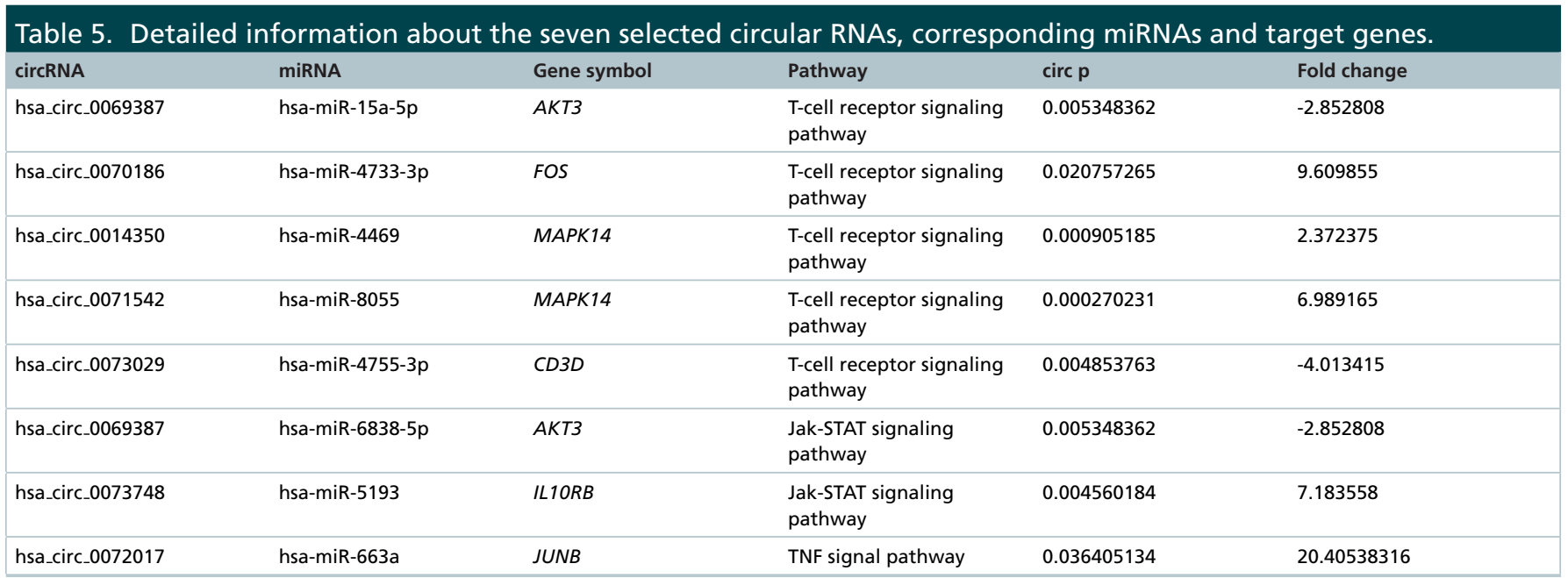

Functional analysis of differentially expressed circRNAs

Functional analysis of differentially expressed circRNAs was undertaken by GO and KEGG pathway enrichment analyses based on the differentially expressed mRNAs predicted to be regulated by circRNAs through 'ceRNA mechanism'. There are three GO categories, including biological process (BP), cell component (CC), and molecular function (MF). The data of GO and KEGG pathway enrichment analyses are shown in Supplementary File 4 and 5. The top ten enriched GO terms (in BP, CC and MF) and top 30 enriched KEGG pathways of the differentially expressed circRNAs are displayed in Figure 4. The three most enriched GO terms ' $T$ cell differentiation in thymus', 'negative regulation of signal transduction' and 'protein repair' in BP; the top three terms in CC were 'nuclear membrane', 'endoplasmic reticulum' and 'Golgi apparatus'. In MF, 'ion binding', 'metal ion binding', and 'cation binding' were the three most enriched GO terms. Meanwhile, KEGG pathways were enriched in 'T-cell receptor 
(A)
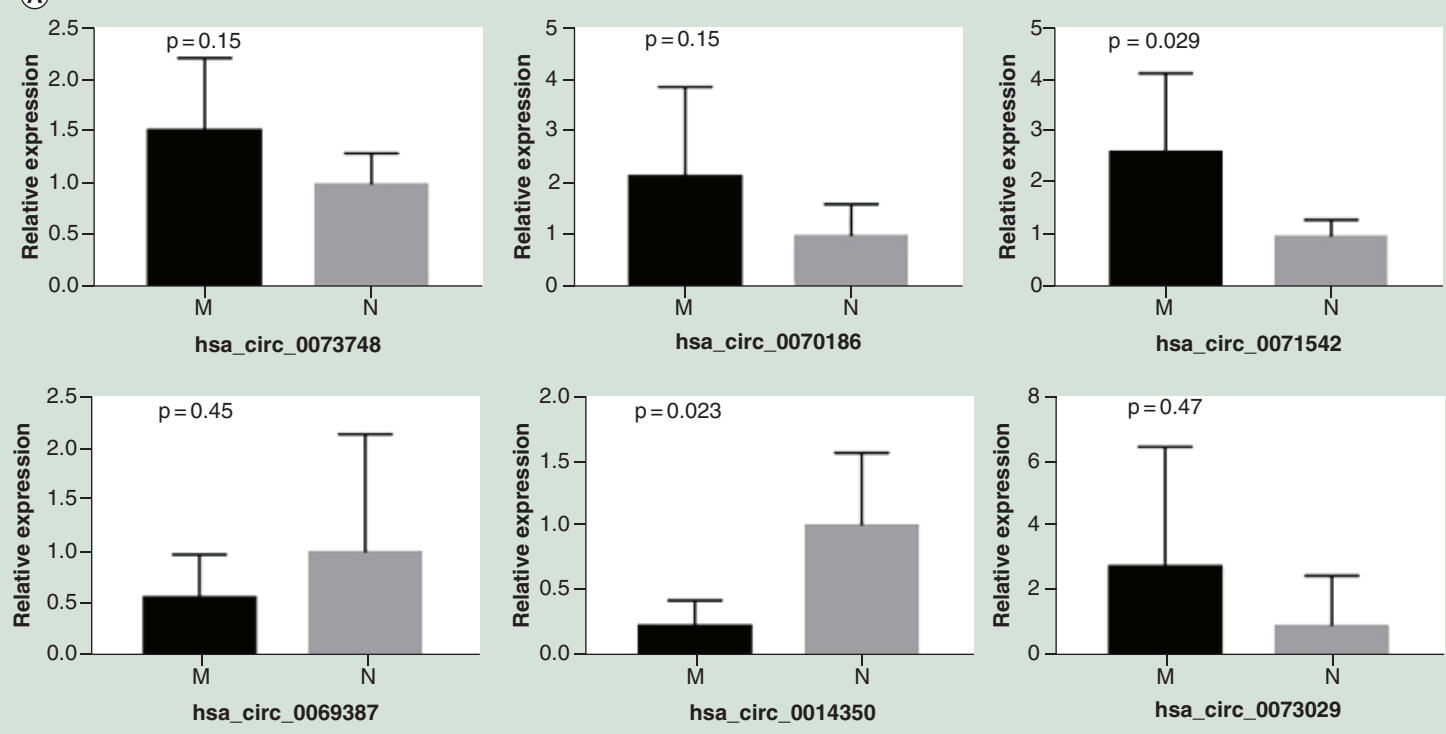

(B)

\section{(C)}

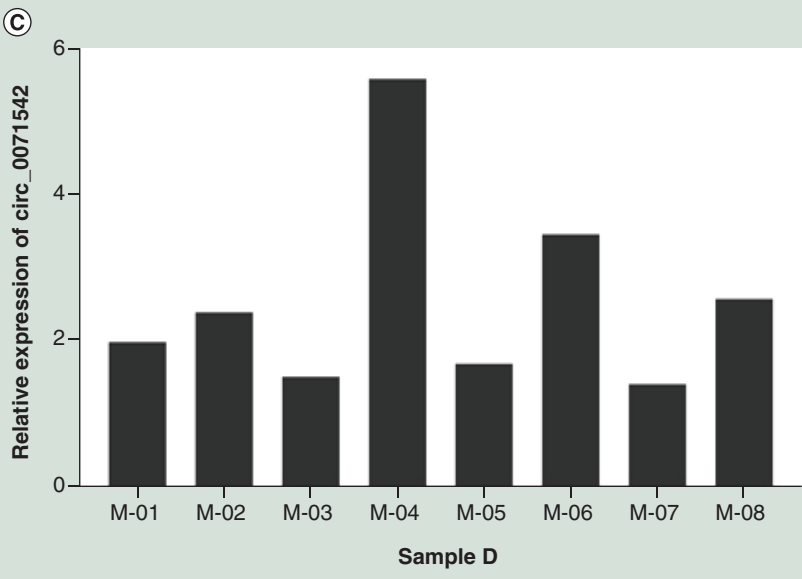

Figure 3. Expression levels of the six selected circular RNAs assessed by quantitative real time-PCR (RT-qPCR). (A) Relative expression levels of the six circRNAs in the fulminant myocarditis group (M) versus control cases (N), assessed by RT-qPCR; data are mean \pm standard deviation. (B) Comparison of mean fold changes (log2 transformed) between RNA microarray data and RT-qPCR results. (C) Relative hsa_circ_0071542 amounts in eight fulminant myocarditis patients. qPCR: Quantitative PCR.

signaling pathway', 'Th1 and Th2 cell differentiation', and 'TNF signaling pathway', which are all associated with inflammation and immunity.

\section{Discussion}

Fulminant myocarditis represents the most serious type of myocarditis, with a rapid onset and dramatically accelerated progression resulting in extremely damaging heart failure, severely altered hemodynamics and cardiogenic shock. The etiology of myocarditis is not completely understood. It is widely believed that genetic and environmental factors cause myocardial damage. Exogenous physical, chemical and microbiological agents might directly promote damage to cardiomyocytes, inducing inflammation. More than $50 \%$ of pediatric acute myocarditis cases result from infection by viruses [24], such as enteroviruses (particularly coxsackieviruses) [25,26], parvovirus B19 [27] and human herpesvirus type 6 [28]. Compared with viruses, bacteria and other infectious agents, such as protozoa, fungi and parasites, are known to cause myocarditis far less commonly [29]. Drugs, particularly chemotherapeutics, may also cause myocardial inflammation via direct cardiotoxic effects or by inducing hypersensitivity [30]. The myocardium could equally be injured by endogenous molecules which induce inflammation [31]. In addition, 


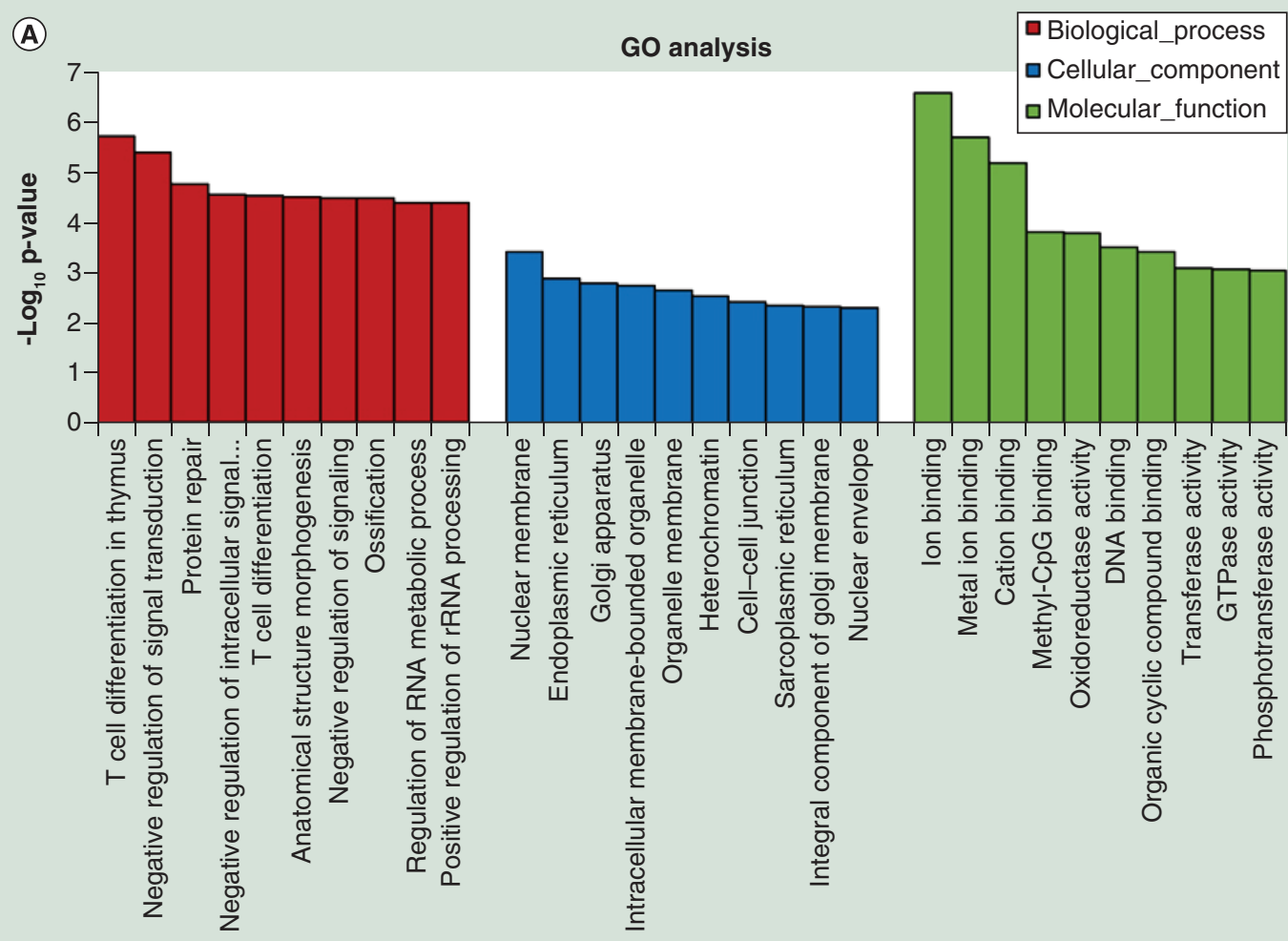

(B)

(3)

Top 30 of pathway enrichment

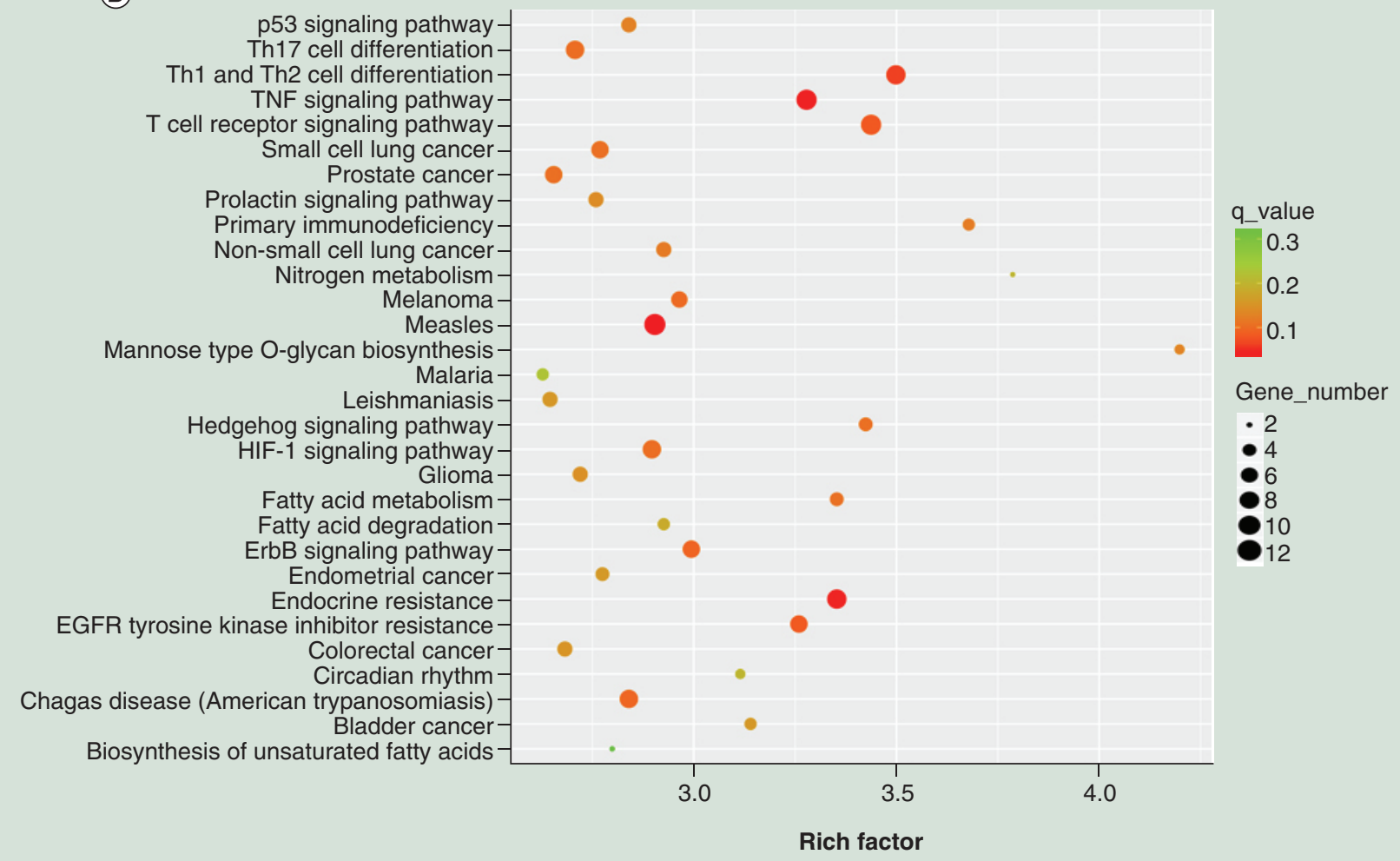

Figure 4. Gene ontology and Kyoto encyclopedia of genes and genomes pathway analyses of dysregulated circular RNA (circRNA)s in fulminant myocarditis. (A) Ten most enriched gene ontology terms in biological process, Cellular component, and molecular function for differentially expressed circRNAs. (B) Top 30 Kyoto encyclopedia of gene and genome pathways of dysregulated circRNAs. circRNA: circular RNA: EGFR: Epidermal growth factor receptor; GO: Gene ontology. 
genetic and epigenetic factors have been reported to play critical roles in myocarditis by regulating inflammation and immune reactions $[32,33]$.

circRNAs are considered critical modulators in a number of diseases [34], including cardiovascular disease [16,35]. Moreover, circRNAs are known to regulate immune and inflammatory reactions [36,37]. Nevertheless, the expression profiles and functions of circRNAs in fulminant myocarditis remain elusive.

Here, we first investigated the expression patterns of circRNAs in leukocytes separated from peripheral blood samples of three children with fulminant myocarditis and three healthy volunteers. As shown above, 3173 circRNAs had differential expression (fold change $>2, \mathrm{p}<0.05$ ), including 2281 upregulated and 892 downregulated. This is the first report assessing the expression patterns of circRNAs in fulminant myocarditis, which may provide new insights into circRNA functions in this disease. Next, seven differentially expressed circRNAs were selected for microarray data validation by RT-qPCR. Among them, circ_0071542 was confirmed to be upregulated in fulminant myocarditis cases versus control patients. Relative circ_0071542 amounts were different in the eight fulminant myocarditis cases assessed, with patient M-04 displaying the highest expression. It should be noted that patient M-04 died from heart failure and multiple organ failure and represented the most serious case. This finding indicates that circ_0071542 expression may be associated with the severity of fulminant myocarditis.

Our data revealed all 3173 dysregulated circRNAs possessed miRNA binding sites. Circ_0071542 was predicted to regulate the expression of $M A P K$ by binding hsa-miR-8055. A number of scholars have demonstrated that $M A P K$ participates in the development of myocarditis [38-40]. Thus, we speculated that circ_0071542 has a proinflammatory function in fulminant myocarditis by sponging miR-8055 to control MAPK expression. However, the associations of circ_0071542 and miR-8055 with $M A P K$ in fulminant myocarditis require further investigation.

GO and KEGG pathway enrichment analyses were conducted to preliminarily study the roles and potential mechanisms of dysregulated circRNAs in the development of fulminant myocarditis. The three most enriched GO terms included 'T cell differentiation in thymus', 'ion binding', and 'metal ion binding'. KEGG pathways were enriched 'T-cell receptor signaling pathway', 'Th1 and Th2 cell differentiation', and 'TNF signaling pathway'. These pathways all contribute to inflammation and immunity [21,41]. To the best of our knowledge, the pathogenesis of fulminant myocarditis is associated with the immune response and overwhelming inflammatory reactions elicited by the pathogen. Thus, the current findings suggested the above differentially expressed circRNAs might contribute to fulminant myocarditis via these pathways.

\section{Conclusion}

Overall, this study first provided a comprehensive expression profile of differentially expressed circRNAs in children with fulminant myocarditis. GO and KEGG pathway enrichment analyses and circRNA-miRNA-mRNA interaction network building were conducted to preliminarily assess the roles and potential mechanisms of dysregulated circRNAs in the development of fulminant myocarditis. Hsa_circ_0071542 was confirmed to be markedly upregulated in fulminant myocarditis, suggesting it may constitute a candidate biomarker of fulminant myocarditis. Altogether, this work may provide a novel theoretical basis for further studies on circRNAs in fulminant myocarditis. The limitations of the present study should be mentioned. First, it had a small sample size due to the low incidence of fulminant myocarditis and the lack of myocardial biopsy. In microarray analysis, the smaller the sample size, the higher the false positive rate. This may be one of the reasons why the current study had a low verification rate (one in seven) by RT-qPCR. The upregulated trend of circ_0071542 was verified by RT-qPCR at least five-times and would be consistent for an elevated number of patients. However, the fold change may change due to varying severity in different cases. It is well known that myocardial biopsy is the gold standard for myocarditis diagnosis. Myocardial biopsy can directly reveal the severity of myocardial damage, so the relationship between circ_0071542 expression and the severity of myocardial damage can be verified. Further multicenter studies with larger sample sizes should be carried out, as well as circRNA knockdown or overexpression experiments, to reveal the functions and mechanisms of these circRNAs in children with fulminant myocarditis.

\section{Future perspective}

Fulminant myocarditis represents the most serious type of myocarditis. It is very challenging to promptly diagnose myocarditis in pediatric cases, as clinical manifestations range from no myocardial dysfunction to sudden cardiac death. Emerging evidences indicate that circRNAs are associated with different cardiovascular diseases and regulate immunity and inflammation. This study provides a new basis for substantial roles of circRNAs in fulminant 
myocarditis. The massive data can be further exploited by other researchers. We hope that hsa_circ_0071542 could be verified in additional clinical samples and serve as a biomarker of fulminant myocarditis.

\section{Summary points}

- Circular RNAs (circRNAs), as newly described noncoding RNAs, are considered imperative modulators in multiple diseases.

- circRNAs act as competitive endogenous RNAs of miRNAs via their miRNA response elements.

- For the first time, this work provided a holistic expression profile of differentially expressed circRNAs in children with fulminant myocarditis.

- In all, 3173 differentially expressed circRNAs were found.

- All 3173 dysregulated circRNAs were predicted with miRNA binding sites, and a circRNA-miRNA-mRNA interaction network was generated.

- GO and KEGG enrichment analyses revealed these dysregulated circRNAs mostly regulate the progression of inflammation and immunity.

- Hsa_circ_0071542 was confirmed to be upregulated by 2.5-fold in fulminant myocarditis patients compared with the control group. Circ_0071542 was predicted to regulate the expression of MAPK by binding hsa-miR-8055.

\section{Supplementary data}

To view the supplementary data that accompany this paper please visit the journal website at: www.futuremedicine.com/doi/sup $\mathrm{pl} / 10.2217 /$ epi-2019-0101

Author contributions

B Han and L Zhang conceived and supervised the study. L Zhang and J Wang designed experiments. L Zhang, Q Liu and Y Kong performed experiments. D Jiang and $\mathrm{H}$ Jia analyzed data. L Zhang wrote the manuscript; Bo Han and Jing Wang made manuscript revisions. All authors reviewed the results and approved the final version of the manuscript.

\section{Financial \& competing interests disclosure}

This study was supported by the National Natural Science Foundation of China (grant number 8187020860); Jinan Science and Technology Development Plan (grant number 201805020); Special Expert of Taishan Scholars (grant number ts201511099). The authors have no other relevant affiliations or financial involvement with any organization or entity with a financial interest in or financial conflict with the subject matter or materials discussed in the manuscript apart from those disclosed.

No funded writing assistance was utilized in the production of this manuscript.

\section{Ethical disclosure}

The authors state that they have obtained appropriate institutional review board approval and have followed the principles outlined in the Declaration of Helsinki for all human participants. In addition, for investigations involving human subjects, informed consent has been obtained from the participants involved.

\section{Open access}

This work is licensed under the Attribution-NonCommercial-NoDerivatives 4.0 Unported License. To view a copy of this license, visit http://creativecommons.org/licenses/by-nc-nd/4.0/

\section{References}

Papers of special note have been highlighted as: $\bullet$ of interest; $\bullet \bullet$ of considerable interest

1. Farinha IT, Miranda JO. Myocarditis in paediatric patients: unveiling the progression to dilated cardiomyopathy and heart failure. J. Cardiovasc. Dev. Dis. 3(4), pii:E31 (2016).

2. Ginsberg F, Parrillo JE. Fulminant myocarditis. Crit. Care Clin. 29(3), 465-483 (2013).

- Reviewed the epidemiology, pathogenesis, diagnosis and management of fulminant myocarditis.

3. Wang D, Jiang J, Li S et al. Chinese society of cardiology expert consensus statement on the diagnosis and treatment of adult fulminant myocarditis. Sci. China. Life Sci. 62(2), 187202 (2018).

4. Maisch B, Ruppert V, Pankuweit S. Management of fulminant myocarditis: a diagnosis in search of its etiology but with therapeutic options. Curr. Heart. Fail. Rep. 11(2), 166-177 (2014).

5. Caforio ALP, Malipiero G, Marcolongo R, Iliceto S. Myocarditis: a clinical overview. Curr. Cardiol. Rep. 19(7), 63 (2017). 
6. Di Filippo S. Improving outcomes of acute myocarditis in children. Expert. Rev. Cardiovasc. Ther. 14(1), 117-125 (2016).

7. Vicens Q, Westhof E. Biogenesis of circular RNAs. Cell 159(1), 13-14 (2014).

8. Qu S, Yang X, Li X et al. Circular RNA: a new star of noncoding RNAs. Cancer Lett. 365(2), 141-148 (2015).

9. Wilusz JE, Sharp PA. Molecular biology. A circuitous route to noncoding RNA. Science 340(6131), 440-441 (2013).

10. Li X, Yang L, Chen LL. The biogenesis, functions, and challenges of circular RNAs. Mol. Cell. 71(3), 428-442 (2018).

- Reviewed the biogenesis and functions of circular RNAs (circRNAs).

11. Hansen TB, Jensen TI, Clausen BH et al. Natural RNA circles function as efficient microRNA sponges. Nature 495(7441), 384-388 (2013).

-. Outlined for the first time that circRNAs exert its biological functions by serving as sponges of miRNA.

12. Chang H, Qu J, Wang J, Liang X, Sun W. Circular RNA circ_0026134 regulates non-small cell lung cancer cell proliferation and invasion via sponging miR-1256 and miR-1287. Biomed. Pharmacother. 112, 108743 (2019).

13. Zhou ZB, Du D, Huang GX, Chen A, Zhu L. Circular RNA Atp9b, a competing endogenous RNA, regulates the progression of osteoarthritis by targeting miR-138-5p. Gene 646, 203-209 (2018).

14. Zhang Z, Yang T, Xiao J. Circular RNAs: promising biomarkers for human diseases. EBioMedicine 34, 267-274 (2018).

15. Greene J, Baird AM, Brady L et al. Circular RNAs: biogenesis, function and role in human diseases. Front. Mol. Biosci. 4, 38 (2017).

16. Altesha MA, Ni T, Khan A, Liu K, Zheng X. Circular RNA in cardiovascular disease. J. Cell. Physiol. 234(5), 5588-5600 (2018).

- Reviewed the role of circRNA in cardiovascular disease.

17. Holdt LM, Kohlmaier A, Teupser D. Molecular functions and specific roles of circRNAs in the cardiovascular system. Noncoding RNA Res. 3(2), 75-98 (2018).

18. Carrara M, Fuschi P, Ivan C, Martelli F. Circular RNAs: methodological challenges and perspectives in cardiovascular diseases. J. Cell. Mol. Med. 22(11), 5176-5187 (2018).

19. Wang M, Yu F, Wu W et al. Circular RNAs: a novel type of non-coding RNA and their potential implications in antiviral immunity. Int. J. Biol. Sci. 13(12), 1497-1506 (2017).

20. Li X, Liu CX, Xue W et al. Coordinated circRNA biogenesis and function with NF90/NF110 in Viral Infection. Mol. Cell. 67(2), 214-227 (2017).

21. Gaud G, Lesourne R, Love PE. Regulatory mechanisms in T cell receptor signalling. Nat. Rev. Immunol. 18(8), 485-497 (2018).

22. O'shea JJ, Schwartz DM, Villarino AV, Gadina M, Mcinnes IB, Laurence A. The JAK-STAT pathway: impact on human disease and therapeutic intervention. Annu. Rev. Med. 66, 311-328 (2015).

23. Welsch K, Holstein J, Laurence A, Ghoreschi K. Targeting JAK/STAT signalling in inflammatory skin diseases with small molecule inhibitors. Eur. J. Immunol. 47(7), 1096-1107 (2017).

24. Bowles NE, Bowles KR, Towbin JA. Viral genomic detection and outcome in myocarditis. Heart. Fail. Clin. 1(3), 407-417 (2005).

25. Dettmeyer R, Baasner A, Schlamann M, Haag C, Madea B. Coxsackie B3 myocarditis in 4 cases of suspected sudden infant death syndrome: diagnosis by immunohistochemical and molecular-pathologic investigations. Pathol. Res. Pract. 198(10), 689-696 (2002).

26. Mahrholdt H, Wagner A, Deluigi CC et al. Presentation, patterns of myocardial damage, and clinical course of viral myocarditis. Circulation 114(15), 1581-1590 (2006).

27. Molina KM, Garcia X, Denfield SW et al. Parvovirus B19 myocarditis causes significant morbidity and mortality in children. Pediatr. Cardiol. 34(2), 390-397 (2013).

28. Comar M, D'agaro P, Campello C et al. Human herpes virus 6 in archival cardiac tissues from children with idiopathic dilated cardiomyopathy or congenital heart disease. J. Clin. Pathol. 62(1), 80-83 (2009).

29. Cooper LT, Jr. Myocarditis. N. Engl. J. Med. 360(15), 1526-1538 (2009).

30. Durani Y, Giordano K, Goudie BW. Myocarditis and pericarditis in children. Pediatr. Clin. North Am. 57(6), 1281-1303 (2010).

31. Bybee KA, Prasad A. Stress-related cardiomyopathy syndromes. Circulation 118(4), 397-409 (2008).

32. Campuzano O, Fernández-Falgueras A, Sarquella-Brugada G et al. A genetically vulnerable myocardium may predispose to myocarditis. J. Am. Coll. Cardiol. 66(25), 2913-2914 (2015).

33. Rose NR, Neumann DA, Herskowitz A, Traystman MD, Beisel KW. Genetics of susceptibility to viral myocarditis in mice. Pathol. Immunopathol. Res. 7(4), 266-278 (1988).

34. Han B, Chao J, Yao H. Circular RNA and its mechanisms in disease: from the bench to the clinic. Pharmacol. Ther. 187, 31-44 (2018).

35. Zhou MY, Yang JM, Xiong XD. The emerging landscape of circular RNA in cardiovascular diseases. J. Mol. Cell. Cardiol. 122, 134-139 (2018).

36. Hua Q, Chen Y, Liu Y et al. Circular RNA 0039411 is involved in neodymium oxide-induced inflammation and anti-proliferation in a human bronchial epithelial cell line via sponging miR-93-5p. Toxicol. Sci.170(1), 69-81 (2019). 
37. Zhou Z, Du D, Chen A, Zhu L. Circular RNA expression profile of articular chondrocytes in an IL-1beta-induced mouse model of osteoarthritis. Gene 644, 20-26 (2018).

38. Arumugam S, Thandavarayan RA, Veeraveedu PT et al. Involvement of AMPK and MAPK signaling during the progression of experimental autoimmune myocarditis in rats and its blockade using a novel antioxidant. Exp. Mol. Pathol. 93(2), 183-189 (2012).

39. Niu L, Li C, Wang Z, Xu H, An X. Effects of the MAPK pathway and the expression of CAR in a murine model of viral myocarditis. Exp. Ther. Med. 13(1), 230-234 (2017).

40. Zheng C, Wu SM, Lian H et al. Low-intensity pulsed ultrasound attenuates cardiac inflammation of CVB3-induced viral myocarditis via regulation of caveolin-1 and MAPK pathways. J. Cell. Mol. Med. 23(3), 1963-1975 (2019).

41. Liu H, Li P, Wei $Z$ et al. Regulation of $\mathrm{T}$ cell differentiation and function by epigenetic modification enzymes. Semin. Immunopathol. $413315-326$ (2019). 
